\title{
EFFECT OF INITIAL STRESS AND GRAVITY FIELD ON MICROPOLAR THERMOELASTIC SOLID WITH MICROTEMPERATURES
}

\author{
Mohamed I.A. Othman \\ Zagazig University, Department of Mathematics, Faculty of Science, Zagazig, Egypt \\ Taif University, Department of Mathematics, Faculty of Science, Taif, Saudi Arabia \\ e-mail:m_i_a_othman@yahoo.com
}

RAMADAN S. TANTAWI

Zagazig University, Department of Mathematics, Faculty of Science, Zagazig, Egypt e-mail:ramadan_tantawi1@yahoo.com

Mohamed I.M. HiLAL

Zagazig University, Department of Mathematics, Faculty of Science, Zagazig, Egypt

Sinai University, Department of Basic Sciences, Faculty of Engineering Sciences, El-Arish, Egypt

e-mail:mimhilal@yahoo.com

\begin{abstract}
The purpose of the present article is the study of the effect of the gravity field on an initially stressed micropolar thermoelastic medium with microtemperatures. The analytical method used to obtain the formula of the physical quantities is the normal mode analysis. The comparisons are established graphically in the presence and the absence of gravity, initial stress and micropolar thermoelasticity. The main conclusions state that the gravity, initial stress and the micropolar thermoelasticity are effective physical operators on the variation of the physical quantities. The microtemperatures are very useful theory in the field of geophysics and earthquake engineering.
\end{abstract}

Keywords: gravity, initial stress, micropolar thermoelasticity, microtemperatures

\section{Introduction}

The theory of elastic micropolar materials was introduced by Eringen (1966). The theory of continuum micropolar mechanics takes into consideration the microstructure of materials. Description of the micropolar materials is useful for fibrous, lattice or, in general, materials having microstructural construction having in each point extra rotational degrees of freedom independent of translation. The material, however, can transmit couple stress. Smith (1967) studied wave propagation in micropolar elastic solids. Parfitt and Eringen (1971) investigated reflection of plane waves from a flat boundary of a micropolar elastic half-space. Ariman (1972) also studied wave propagation in a micropolar elastic half-space solid. Eringen (1999) presented the microcontinuum field theory. Kumar and Ailawalia (2005) studied the response of a micropolar cubic crystal due to various sources. Kumar and Gupta (2010) studied propagation of waves in a transversely isotropic micropolar generalized thermoelastic half-space. Abbas and Kumar (2013) studied deformation due to a thermal source in micropolar thermoelastic media with the two-temperature effect. Recently, Othman et al. (2014) established the effect of rotation on a micropolar thermo-elastic solid with two temperatures. Abouelregal and Zenkour (2015) studied a thermoelastic problem of an axially moving micro beam subjected to an external transverse excitation. The concept of microtemperatures means that microelements of a thermoelastic body have different temperatures and depend homogeneously on microcoordinates of the microelements, which are based on the 
microstructure of the continuum. Grot (1969) established the thermodynamic theory of elastic materials with inner structures, in which microdeformations and particles possess microtemperatures. Eringen and Kafadar (1976) presented the basis for the microelements with microtemperatures. Riha (1979) presented a study of heat conduction in materials with inner structures. Iesan and Quintanilla (2000) constructed the linear theory of thermoelasticity for materials with inner structure whose particles, in addition to the classical displacement and temperature fields, possess microtemperatures. Iesan $(2001,2004)$ presented the mathematical model of theory of micromorphic elastic solids with microtemperatures, in which microelements possess microtemperatures and can stretch and contract independently of their translations. Casas and Quintanilla (2005) studied exponential stability in thermoelasticity with microtemperatures. Scalia and Svanadze (2006) discussed solutions of the theory of thermoelasticity with microtemperatures. Iesan $(2006,2007)$ presented a study of thermoelastic bodies with a microstructure and microtemperatures.

The effect of gravity on wave propagation in an elastic medium was first considered by Bromwich (1898) who treated the force of gravity as a type of a body force. Love (1965) extended the work of Bromwich investigating the influence of gravity on superficial waves and showed that the Rayleigh wave velocity is affected by the gravitational field. Sezawa (1927) studied dispersion of elastic waves propagating on curved surfaces. Othman et al. (2013a,b) investigated two models on the effect of the gravitational field on thermoelastic solids. The presence of initial stresses in solid materials has a substantial effect on their subsequent response to applied loads that is very different from the corresponding response in the absence of initial stresses. In geophysics, as an example, high stress developed below the Earth's surface due to gravity has a strong influence on the propagation speed of elastic waves. While in soft biological tissues initial (or residual), stresses in artery walls ensure that the circumferential stress distribution through thickness of the artery wall is close to uniform at typical physiological blood pressures. Initial stresses may arise, for example, from applying loads, as in the case of gravity, processes of growth and development in living tissue or, in the case of engineering components, from the manufacturing process. Ames and Straughan (1999) derived continuous dependence results for initially pre-stressed thermoelastic bodies. Montanaro (1999) investigated isotropic linear thermoelasticity with hydrostatic initial stress. Wang and Slattery (2002) formulated thermoelastic equations without energy dissipation for initially stressed bodies. Iesan (2008) presented a theory of Cosserat thermoelastic solids with initial stresses. Recently, Othman et al. (2015) discussed the effect of initial stress on a thermoelastic rotating medium with laser pulse heating.

This investigation studies the 2D problem of linear, isotropic, homogeneous initially stressed micropolar thermoelastic solid influenced by the gravity field. The application of the present model cannot be ignored in geophysics and earthquake engineering due to the importance of the microtemperature properties. The normal mode analysis is the analytical method used to obtain the solutions of the considered physical quantities which are graphically represented in the absence and presence of the studied physical effects.

\section{Basic equations}

Consider the linear theory of thermodynamics for isotropic elastic materials with inner structure. According to Eringen (1999), Isean (2007) and Montanaro (1999), the field equations and the constitutive relations for a linear, homogeneous, isotropic initially stressed micropolar thermoelastic solid with microtemperatures without body forces, body couples, heat sources and first heat source moment, can be considered as 


$$
\begin{aligned}
& \frac{\partial \sigma_{i j}}{\partial x_{i}}=\rho \frac{\partial^{2} u_{j}}{\partial t^{2}} \quad \frac{\partial m_{i j}}{\partial x_{i}}+\varepsilon_{i j r} \sigma_{i r}-\mu_{1} \frac{\partial w_{j}}{\partial x_{i}}=J \rho \frac{\partial^{2} \phi_{j}}{\partial t^{2}} \\
& k_{6} \frac{\partial^{2} w_{i}}{\partial x_{j}^{2}}+\left(k_{4}+k_{5}\right) \frac{\partial^{2} w_{j}}{\partial x_{i} \partial x_{j}}+\mu_{1} \frac{\partial}{\partial t} \frac{\partial \phi_{j}}{\partial x_{i}}-k_{2} w_{i}-b \frac{\partial w_{i}}{\partial t}-k_{3} \frac{\partial T}{\partial x_{i}}=0 \\
& k \frac{\partial^{2} T}{\partial x_{i}^{2}}-\rho C_{e} \frac{\partial T}{\partial t}-\gamma_{1} T_{0} \frac{\partial}{\partial t} \frac{\partial u_{i}}{\partial x_{i}}+k_{1} \frac{\partial w_{i}}{\partial x_{i}}=0
\end{aligned}
$$

and

$$
\begin{array}{rlrl}
\sigma_{i j} & =\lambda \frac{\partial u_{r}}{\partial x_{r}} \delta_{i j}+\mu\left(\frac{\partial u_{i}}{\partial x_{j}}+\frac{\partial u_{j}}{\partial x_{i}}\right)+k^{*}\left(\frac{\partial u_{i}}{\partial x_{j}}-\varepsilon_{i j r} \phi_{r}\right)-\gamma_{1} T \delta_{i j}-p\left(\delta_{i j}+\omega_{i j}\right) \\
m_{i j} & =\alpha \frac{\partial \phi_{r}}{\partial x_{r}} \delta_{i j}+\beta \frac{\partial \phi_{i}}{\partial x_{j}}+\gamma \frac{\partial \phi_{j}}{\partial x_{i}} & q_{i} & =k \frac{\partial T}{\partial x_{i}}+k_{1} w_{i} \\
q_{i j} & =-k_{4} \frac{\partial w_{k}}{\partial x_{k}} \delta_{i j}-k_{5} \frac{\partial w_{i}}{\partial x_{j}}-k_{6} \frac{\partial w_{j}}{\partial x_{i}} & Q_{i} & =\left(k_{1}-k_{2}\right) w_{i}+\left(k-k_{3}\right) \frac{\partial T}{\partial x_{i}} \\
e_{i j} & =\frac{1}{2}\left(\frac{\partial u_{i}}{\partial x_{j}}+\frac{\partial u_{j}}{\partial x_{i}}\right) & \omega_{i j} & =\frac{1}{2}\left(\frac{\partial u_{j}}{\partial x_{i}}-\frac{\partial u_{i}}{\partial x_{j}}\right)
\end{array}
$$

where $\lambda$ and $\mu$ are Lamé constants, $\alpha, \beta, \gamma$, and $k^{*}$ are micropolar constants, $\gamma_{1}=\left(3 \lambda+2 \mu+k^{*}\right) \alpha_{t}$, while $\alpha_{t}$ is the linear thermal expansion coefficient, $\rho$ is density, $C_{e}$ - specific heat, $k$ - thermal conductivity, $u_{i}$ - displacement vector, $T$ - absolute temperature, $T_{0}$ - reference temperature chosen so that $\left|\left(T-T_{0}\right) / T_{0}\right| \ll 1, \phi_{j}$ is the microrotation vector, $\sigma_{i j}$ are components of stresses, $e_{i j}$ are components of strains, $\delta_{i j}$ is the Kronecker delta, $\varepsilon_{i j r}$ is the permutation symbol, $p-$ pressure, $m_{i j}$ are couple stresses, $J$ is microinertia, $w_{i}$ - microtemperature vector, $\mu_{1}, b, k_{i}$ $(i=1,2, \ldots, 6)$ are constitutive coefficients, $q_{i}$ is heat flux moment, $q_{i j}$ - first heat flux moment and $Q_{i}$ is the mean heat flux vector.

\section{Formulation and solution of the problem}

Consider an isotropic, linear, homogeneous, initially stressed micropolar thermoelastic solid with microtemperatures. Consider also a half-space $(y \geqslant 0)$ and the rectangular Cartesian coordinate system $(x, y, z)$ originated in the surface $z=0$. For a two-dimensional problem, assume the dynamic displacement vector as $u_{i}=(u, v, 0)$. The microrotation vector $\phi_{j}$ will be $\phi_{j}=\left(0,0, \phi_{3}\right)$, consequently the microtemperature vector $w_{i}$ willbe $w_{i}=\left(w_{1}, w_{2}, 0\right)$. All quantities will be a function of the time variable $t$ and coordinates $x$ and $y$. In the equations, comma denotes derivatives with respect to coordinates system.

Equations (2.1) under the effect of the gravitational field can be stated as

$$
\begin{aligned}
& \left(\mu+k^{*}-\frac{p}{2}\right) \nabla^{2} u+\left(\lambda+\mu+\frac{p}{2}\right) \frac{\partial e}{\partial x}+k^{*} \frac{\partial \phi_{3}}{\partial y}-\gamma_{1} \frac{\partial T}{\partial x}+\rho g \frac{\partial v}{\partial x}=\rho \frac{\partial^{2} u}{\partial t^{2}} \\
& \left(\mu+k^{*}-\frac{p}{2}\right) \nabla^{2} v+\left(\lambda+\mu+\frac{p}{2}\right) \frac{\partial e}{\partial y}-k^{*} \frac{\partial \phi_{3}}{\partial x}-\gamma_{1} \frac{\partial T}{\partial y}-\rho g \frac{\partial u}{\partial x}=\rho \frac{\partial^{2} v}{\partial t^{2}} \\
& \gamma \nabla^{2} \phi_{3}-2 k^{*} \phi_{3}+\left(k^{*}-p\right)\left(\frac{\partial v}{\partial x}-\frac{\partial u}{\partial y}\right)-\mu_{1}\left(\frac{\partial w_{2}}{\partial x}-\frac{\partial w_{1}}{\partial y}\right)=J \rho \frac{\partial^{2} \phi_{3}}{\partial t^{2}} \\
& k_{6} \nabla^{2} w_{1}+\left(k_{4}+k_{5}\right) \frac{\partial}{\partial x}\left(\frac{\partial w_{1}}{\partial x}+\frac{\partial w_{2}}{\partial y}\right)+\mu_{1} \frac{\partial}{\partial t} \frac{\partial \phi_{3}}{\partial y}-k_{2} w_{1}-b \frac{\partial w_{1}}{\partial t}-k_{3} \frac{\partial T}{\partial x}=0 \\
& k_{6} \nabla^{2} w_{2}+\left(k_{4}+k_{5}\right) \frac{\partial}{\partial y}\left(\frac{\partial w_{1}}{\partial x}+\frac{\partial w_{2}}{\partial y}\right)-\mu_{1} \frac{\partial}{\partial t} \frac{\partial \phi_{3}}{\partial x}-k_{2} w_{2}-b \frac{\partial w_{2}}{\partial t}-k_{3} \frac{\partial T}{\partial y}=0 \\
& k \nabla^{2} T-\rho C_{e} \frac{\partial T}{\partial t}-\gamma_{1} T_{0} \frac{\partial e}{\partial t}+k_{1}\left(\frac{\partial w_{1}}{\partial x}+\frac{\partial w_{2}}{\partial y}\right)=0
\end{aligned}
$$

where $g$ is the acceleration of gravity and $e$ is dilatation. 
Define non-dimensional variables by expressions

$$
\begin{array}{lll}
x_{i}^{\prime}=\frac{\omega_{1}^{*}}{c_{0}} x_{i} & u_{i}^{\prime}=\frac{\rho c_{0} \omega_{1}^{*}}{\gamma_{1} T_{0}} u_{i} & \phi_{3}^{\prime}=\frac{\rho c_{0}^{2}}{\gamma_{1} T_{0}} \phi_{3} \\
w_{i}^{\prime}=\frac{c_{0}}{\omega_{1}^{*}} w_{i} & m_{i j}^{\prime}=\frac{\omega_{1}^{*}}{\gamma_{1} c_{0} T_{0}} m_{i j} & q_{i j}^{\prime}=\frac{\mu c_{0}^{2}}{\omega_{1}^{*}} q_{i j} \\
\left(T^{\prime}, p_{2}^{\prime}\right)=\frac{1}{T_{0}}\left(T, p_{2}\right) & \left(\sigma_{i j}^{\prime}, p_{1}^{\prime}\right)=\frac{1}{\gamma_{1} T_{0}}\left(\sigma_{i j}, p_{1}\right) & t^{\prime}=\omega_{1}^{*} t \\
g^{\prime}=\frac{g}{c_{0} \omega_{1}^{*}} & \omega_{1}^{*}=\frac{\rho C_{e} c_{0}^{2}}{k} & c_{0}^{2}=\frac{\lambda+2 \mu+k^{*}}{\rho}
\end{array}
$$

Assuming the potential functions $\psi_{1}(x, y, t), \psi_{2}(x, y, t), q_{1}(x, y, t)$ and $q_{2}(x, y, t)$ in dimensionless form, we have

$$
u=\frac{\partial \psi_{1}}{\partial x}+\frac{\partial \psi_{2}}{\partial y} \quad v=\frac{\partial \psi_{1}}{\partial y}-\frac{\partial \psi_{2}}{\partial x} \quad w_{1}=\frac{\partial q_{1}}{\partial x}+\frac{\partial q_{2}}{\partial y} \quad w_{2}=\frac{\partial q_{1}}{\partial y}-\frac{\partial q_{2}}{\partial x}
$$

To get the solution for the physical quantities, consider it in form of the normal mode as

$$
\left[\psi_{1}, \psi_{2}, \phi_{3}, q_{1}, q_{2}, T\right](x, y, t)=\left[\psi_{1}^{*}, \psi_{2}^{*}, \phi_{3}^{*}, q_{1}^{*}, q_{2}^{*}, T^{*}\right](y) \mathrm{e}^{\mathrm{i}(a x-\xi t)}
$$

where $\left[\psi_{1}^{*}, \psi_{2}^{*}, \phi_{3}^{*}, q_{1}^{*}, q_{2}^{*}, T^{*}\right](y)$ are amplitudes of the physical quantities, $\xi$ is the angular frequency, $\mathrm{i}=\sqrt{-1}$ and $a$ is the wave number in the $x$ direction.

Apply equations (3.2)-(3.4) into equations (3.1) and drop the prime to obtain

$$
\begin{aligned}
& {\left[\mathrm{D}^{2}-N_{3}\right] \psi_{1}^{*}-N_{4} \psi_{2}^{*}-N_{5} T^{*}=0 \quad N_{6} \psi_{1}^{*}+\left[\mathrm{D}^{2}-N_{7}\right] \psi_{2}^{*}+c_{2} \phi_{3}^{*}=0} \\
& -c_{6}\left[\mathrm{D}^{2}-a^{2}\right] \psi_{2}^{*}+\left[\mathrm{D}^{2}-N_{8}\right] \phi_{3}^{*}+c_{7}\left[\mathrm{D}^{2}-a^{2}\right] q_{2}^{*}=0 \\
& {\left[\mathrm{D}^{2}-N_{9}\right] q_{1}^{*}-N_{10} T^{*}=0} \\
& N_{11} \phi_{3}^{*}+\left[\mathrm{D}^{2}-N_{12}\right] q_{2}^{*}=0 \\
& N_{14}\left[\mathrm{D}^{2}-a^{2}\right] \psi_{1}^{*}+c_{16}\left[\mathrm{D}^{2}-a^{2}\right] q_{1}^{*}+\left[\mathrm{D}^{2}-N_{13}\right] T^{*}=0
\end{aligned}
$$

where $\mathrm{D}=d / d y$. All the constants are given in Appendix B.

Eliminating $\psi_{1}^{*}, \psi_{2}^{*}, \phi_{3}^{*}, q_{1}^{*}, q_{2}^{*}$ and $T^{*}$ from equations (3.5), enables one to obtain the following differential equations

$$
\left[\mathrm{D}^{12}-\lambda_{1} \mathrm{D}^{10}+\lambda_{2} \mathrm{D}^{8}-\lambda_{3} \mathrm{D}^{6}+\lambda_{4} \mathrm{D}^{4}-\lambda_{5} \mathrm{D}^{2}+\lambda_{6}\right]\left\{\psi_{1}^{*}(y), \psi_{2}^{*}(y), \phi_{3}^{*}(y), q_{1}^{*}(y), q_{2}^{*}(y), T^{*}(y)\right\}=0
$$

where $\lambda_{n}(n=1,2, \ldots, 6)$ are constants.

Equation (3.6) can be factored as

$$
\begin{aligned}
& {\left[\left(\mathrm{D}^{2}-S_{1}^{2}\right)\left(\mathrm{D}^{2}-S_{2}^{2}\right)\left(\mathrm{D}^{2}-S_{3}^{2}\right)\left(\mathrm{D}^{2}-S_{4}^{2}\right)\left(\mathrm{D}^{2}-S_{5}^{2}\right)\left(\mathrm{D}^{2}-S_{6}^{2}\right)\right]} \\
& \quad \cdot\left\{\psi_{1}^{*}(y), \psi_{2}^{*}(y), \phi_{3}^{*}(y), q_{1}^{*}(y), q_{2}^{*}(y), T^{*}(y)\right\}=0
\end{aligned}
$$

where $S_{n}^{2}(n=1,2, \ldots, 6)$ are the roots of the characteristic equation of (3.7).

The general solution to equation (3.7) bounded at $y \rightarrow \infty$ is given by

$$
\begin{array}{ll}
u(x, y, t)=\sum_{n=1}^{6} G_{1 n} R_{n} \mathrm{e}^{-S_{n} y+\mathrm{i}(a x-\xi t)} & v(x, y, t)=\sum_{n=1}^{6} G_{2 n} R_{n} \mathrm{e}^{-S_{n} y+\mathrm{i}(a x-\xi t)} \\
\phi_{3}(x, y, t)=\sum_{n=1}^{6} A_{2 n} R_{n} \mathrm{e}^{-S_{n} y+\mathrm{i}(a x-\xi t)} & T(x, y, t)=\sum_{n=1}^{6} A_{5 n} R_{n} \mathrm{e}^{-S_{n} y+\mathrm{i}(a x-\xi t)} \\
m_{x z}(x, y, t)=\sum_{n=1}^{6} G_{9 n} R_{n} \mathrm{e}^{-S_{n} y+\mathrm{i}(a x-\xi t)} & w_{2}(x, y, t)=\sum_{n=1}^{6} G_{4 n} R_{n} \mathrm{e}^{-S_{n} y+\mathrm{i}(a x-\xi t)} \\
\sigma_{x y}(x, y, t)=\sum_{n=1}^{6} G_{7 n} R_{n} \mathrm{e}^{-S_{n} y+\mathrm{i}(a x-\xi t)} & q_{x y}(x, y, t)=\sum_{n=1}^{6} G_{12 n} R_{n} \mathrm{e}^{-S_{n} y+\mathrm{i}(a x-\xi t)}
\end{array}
$$

Here $R_{n}(n=1,2, \ldots, 6)$ are some coefficients. The other field quantities are given in Appen$\operatorname{dix} \mathrm{A}$. 


\section{Applications}

Consider the following non-dimensional boundary conditions to determine the coefficients $R_{n}$ $(n=1,2, \ldots, 6)$ and neglect the positive exponentials to avoid unbounded solutions at infinity. The surface of the medium satisfies the following conditions $y=0$ :

- The mechanical boundary conditions are

- normal stress condition (mechanically stressed by the constant force $p_{1}$ ), so that

$$
\sigma_{y y}=-p_{1} \mathrm{e}^{\mathrm{i}(a x-\xi t)}-p
$$

- tangential stress condition (stress free)

$$
\sigma_{x y}=0
$$

- Condition of couple stress (couple stress is constant in the $y$-direction) implying that

$$
m_{x z}=0
$$

- Thermal condition (half-space subjected to thermal shock with constant temperature $p_{2}$ applied to the boundary) leading to

$$
T=p_{2} \mathrm{e}^{\mathrm{i}(a x-\xi t)}
$$

- Normal and tangential heat flux moments are free, so that

$$
q_{y y}=q_{x y}=0
$$

Substituting the expressions of the considered quantities into boundary conditions (4.1)-(4.5), one obtains equations satisfied by the coefficients $R_{n}(n=1,2, \ldots, 6)$. Applying the inverse of matrix method to the raised system of equations, one finds values of the coefficients $R_{n}$ $(n=1,2, \ldots, 6)$ as

$$
\left[\begin{array}{l}
R_{1} \\
R_{2} \\
R_{3} \\
R_{4} \\
R_{5} \\
R_{6}
\end{array}\right]=\left[\begin{array}{cccccc}
G_{61} & G_{62} & G_{63} & G_{64} & G_{65} & G_{66} \\
G_{71} & G_{72} & G_{73} & G_{74} & G_{75} & G_{76} \\
G_{91} & G_{92} & G_{93} & G_{94} & G_{95} & G_{96} \\
A_{51} & A_{52} & A_{53} & A_{54} & A_{55} & A_{56} \\
G_{111} & G_{112} & G_{113} & G_{114} & G_{115} & G_{116} \\
G_{121} & G_{122} & G_{123} & G_{124} & G_{125} & G_{126}
\end{array}\right]^{-1}\left[\begin{array}{c}
-p_{1} \\
0 \\
0 \\
p_{2} \\
0 \\
0
\end{array}\right]
$$

Thus, we obtain expressions for the physical quantities of the plate surface.

\section{Particular cases}

In the present study, we consider the following particular cases:

(i) Absence of gravity by taking $g=0$ in equations (4.1) and (4.2).

(ii) Non-initial stress effect by taking $p=0$ in equation (4.5).

(iii) Absence of micropolar by taking $\alpha, \beta, \gamma, k^{*}$ and $j=0$ in equations (4.1)-(4.5). 


\section{Numerical results and discussion}

In order to illustrate the obtained theoretical results in the preceding Section, according to Eringen (1984), the magnesium crystal-like thermoelastic micropolar material has been chosen for the purpose of calculations. The used parameters are given in SI units. The constants are taken as $\lambda=9.4 \cdot 10^{10} \mathrm{~N} / \mathrm{m}^{2}, \mu=4 \cdot 10^{10} \mathrm{~N} / \mathrm{m}^{2}, k=1.7 \cdot 10^{2} \mathrm{~N} /(\mathrm{s} \mathrm{K}), \rho=1.74 \cdot 10^{3} \mathrm{~kg} / \mathrm{m}^{3}$, $\alpha_{t}=7.4033 \cdot 10^{-7} / \mathrm{K}, C_{e}=1.04 \cdot 10^{3} \mathrm{~J} /(\mathrm{kg} \mathrm{K}), k *=1 \cdot 10^{10} \mathrm{~N} / \mathrm{m}^{2}, \gamma=7.779 \cdot 10^{-8} \mathrm{~N}$, $J=2 \cdot 10^{-20} \mathrm{~m}^{2}, T_{0}=298 \mathrm{~K}, k_{1}=0.0035 \mathrm{~N} / \mathrm{s}, k_{2}=0.0045 \mathrm{~N} / \mathrm{s}, k_{3}=0.0055 \mathrm{~N} /(\mathrm{s} \mathrm{K})$, $k_{4}=0.065 \mathrm{~N} /\left(\mathrm{s} \mathrm{m}^{2}\right), k_{5}=0.076 \mathrm{~N} /\left(\mathrm{s} \mathrm{m}^{2}\right), k_{6}=0.096 \mathrm{~N} /\left(\mathrm{s} \mathrm{m}^{2}\right), \mu_{1}=0.0085 \mathrm{~N}, b=0.15 \cdot 10^{-9} \mathrm{~N}$, $p_{1}=1 \mathrm{~N} / \mathrm{m}^{2}, p_{2}=2 \mathrm{~K}, a=1.5 \mathrm{~m}, t=0.5 \mathrm{~s}, \xi=\eta+i \eta_{1}, \eta=0.9 \mathrm{rad} / \mathrm{s}, \eta_{1}=2.9 \mathrm{rad} / \mathrm{s}, x=0.5 \mathrm{~m}$, $0 \leqslant y \leqslant 6 \mathrm{~m}$.

The variation of real parts of each displacement $v$, microtemperature vector $w_{2}$, temperature $T$, stress $\sigma_{x y}$, couple stress $m_{x z}$, microrotation $\phi_{3}$ and the first heat flux moment $q_{x y}$ are obtained and represented by the distance $y$.

Figures 1-3 represent the behavior of these physical quantities against the distance $y$ in 2D when $p=5 \mathrm{~N} / \mathrm{m}$ and $g=9.8 \mathrm{~m} / \mathrm{s}^{2}$. Figures $4 \mathrm{a}$ and $4 \mathrm{~b}$ show the behavior of these physical quantities against the distance $y$ in $2 \mathrm{D}$ for $g=9.8 \mathrm{~m} / \mathrm{s}^{2}$ in the case of $p=5 \mathrm{~N} / \mathrm{m}$. Figures $5 \mathrm{a}$ and $5 \mathrm{~b}$ depict the variation of these physical quantities against the distance $y$ in $2 \mathrm{D}$ in the case of presence and absence of micropolar thermoelasticity when the gravity and the initial stress are present.

(a)

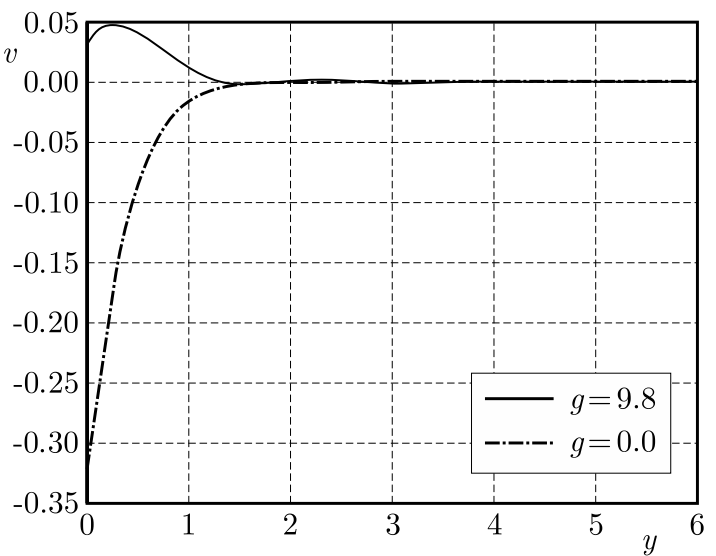

(b)

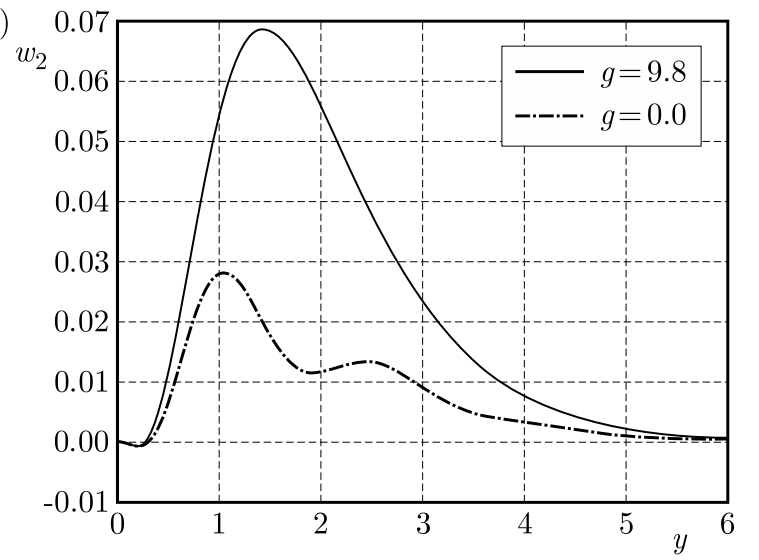

Fig. 1. Variation of displacement $v$ (a) and of microtemperature vector $w_{2}$ (b) against $y$

(a)

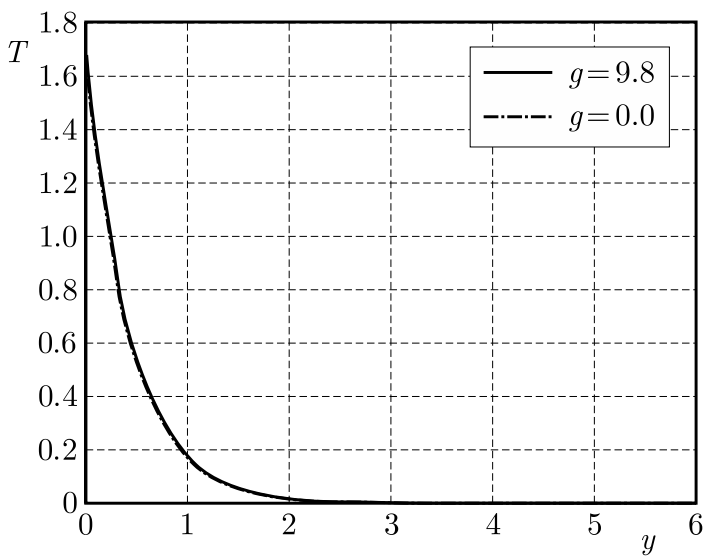

(b)

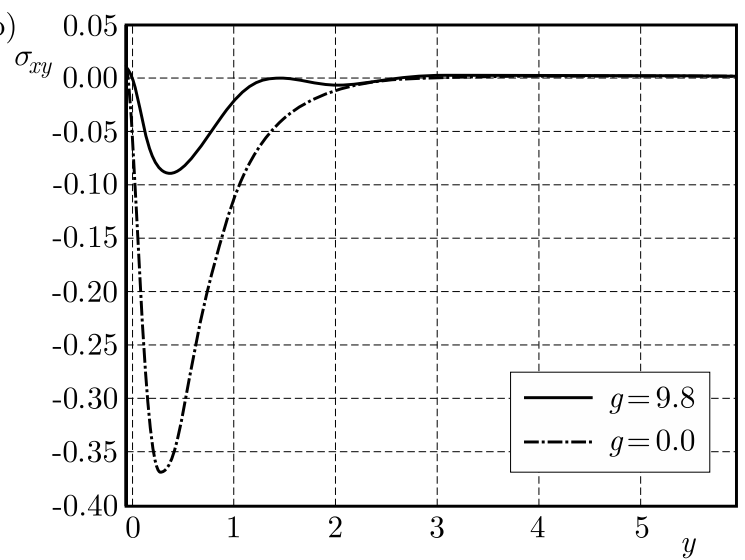

Fig. 2. Variation of temperature $T$ (a) and of stress $\sigma_{x y}$ (b) against $y$ 
(a)

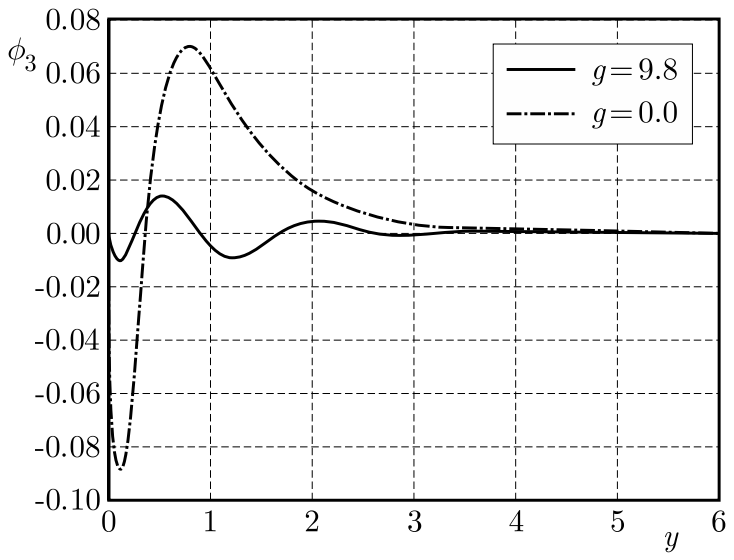

(b)

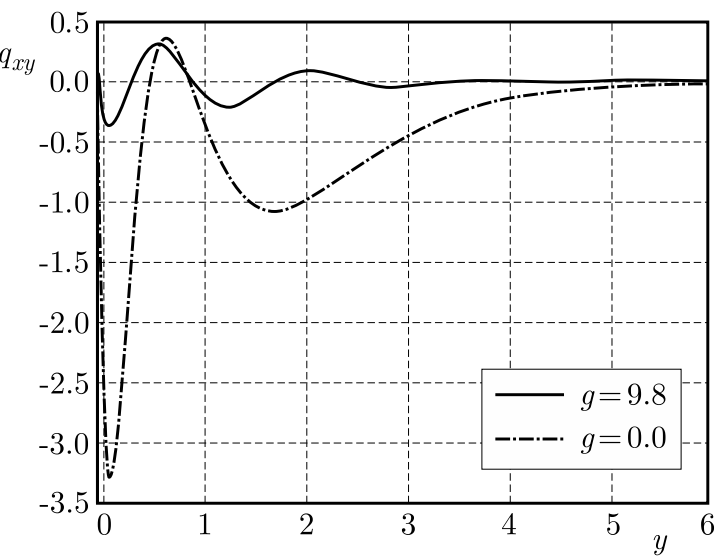

Fig. 3. Variation of microrotation vector $\phi_{3}$ (a) and of the first heat flux moment $q_{x y}$ (b) against $y$

Figure 1a shows that the variation of the displacement component $v$ increases with an increase in gravity for $y \geqslant 0$. Figure $1 \mathrm{~b}$ clarifies the variation of the microtemperature vector $w_{2}$ which decreases with an increase in gravity for $y \geqslant 0$. It is clear from Fig. 2a that the variation of temperature $T$ decreases with an increase in gravity for $y \geqslant 0$, but for very small values it seems to be identical. This means that the effect of gravity has a small influence on the variation of temperature. Figure $2 \mathrm{~b}$ depicts the variation of the shearing stress $\sigma_{x y}$ which increases with an increase in gravity for $y \geqslant 0$. Figure 3 a explains that the variation of the microrotation vector $\phi_{3}$ increases in the interval $0 \leqslant y \leqslant 0.5$, while it decreases in the interval $0.5 \leqslant y \leqslant 6$, with an increase in gravity. Figure $3 \mathrm{~b}$ determines the variation of the heat flux moment $q_{x y}$ which increases at the intervals $0 \leqslant y \leqslant 0.6$ and $1 \leqslant y \leqslant 6$, but decreases at the interval $0.6 \leqslant y \leqslant 1$ with an increase in gravity. The gravity has an effective role in the variation of all physical quantities of the problem. One can notice a change in the variation of the physical quantities while gravity is present or absent.

Figure 4 a shows that the variation of the displacement component $v$ decreases in the intervals $0 \leqslant y \leqslant 0.4,1 \leqslant y \leqslant 1.8$ and $2.8 \leqslant y \leqslant 6$, while it increases in the intervals $0.4 \leqslant y \leqslant 1$ and $1.8 \leqslant y \leqslant 2.8$ with an increase in the initial stress. Figure $4 \mathrm{~b}$ clarifies the variation of the microtemperature vector $w_{2}$ which increases with an increase in the initial stress for $y \geqslant 0$. It is clear that all functions are continuous and all the curves converge to zero. The initial stress has a significant role in the variation of all physical quantities in the problem. This can be deduced from changing of the manner of variation of the physical quantities while the effect of the initial stress is present or absent.

(a)

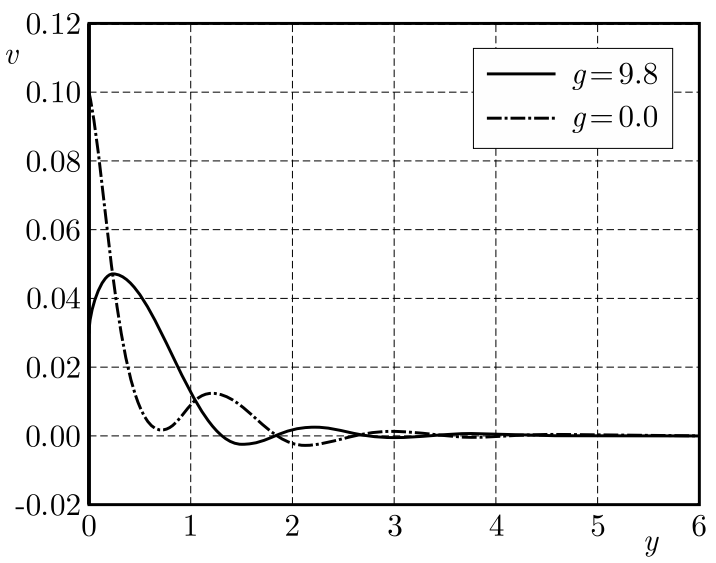

(b)

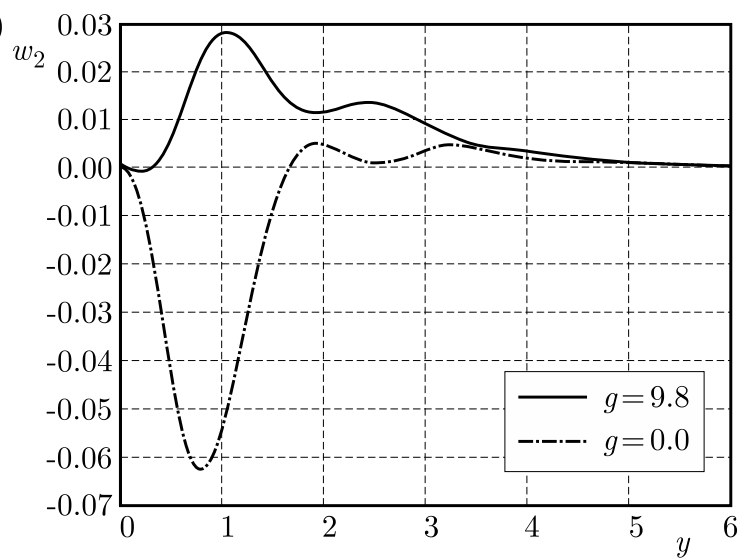

Fig. 4. Variation of displacement $v$ (a) and of microtemperature vector $w_{2}$ (b) against $y$ 
Figure 5a shows that the variation of the displacement component $v$ increases in the intervals $0 \leqslant y \leqslant 2$ and $4.4 \leqslant y \leqslant 6$, while it decreases in the interval $2 \leqslant y \leqslant 4.4$ with an increase in the micropolar thermoelasticity. It is clear from Fig. $5 \mathrm{~b}$ that the variation of temperature $T$ decreases with an increase of the micropolar thermoelasticity for $y \geqslant 0$ in observable behavior. It is clear that all functions are continuous and all the curves converge to zero. The micropolar thermoelasticity plays an important role in the variation of all physical quantities in the problem. The micropolar thermoelasticity is a very important property in thermoelastic materials with a microstructure.

(a)

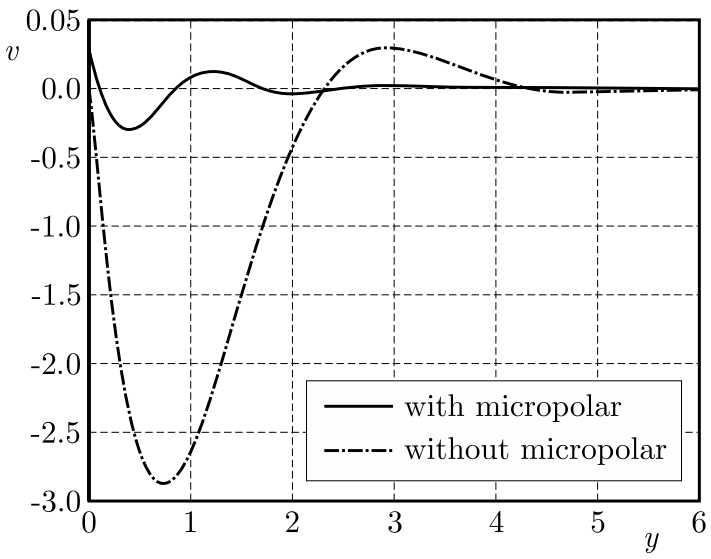

(b)

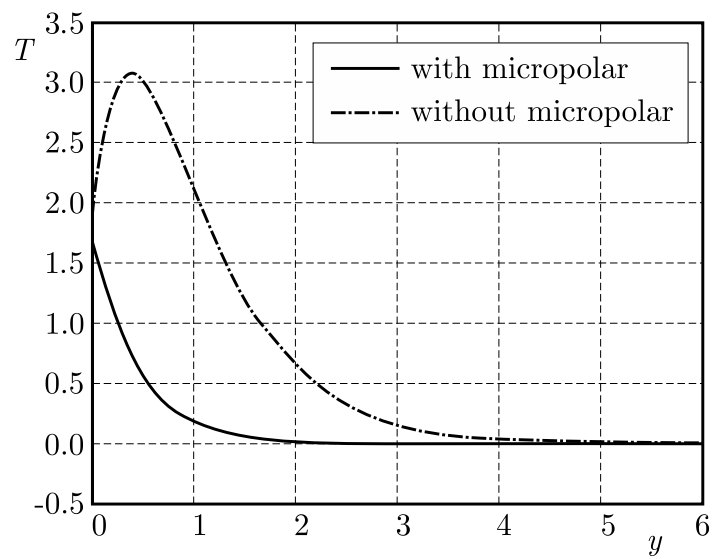

Fig. 5. Variation of displacement $v$ (a) and of temperature $T$ (b) against $y$ with and without micropolar

The 3D curves of the quantities $v$ and $w_{1}$ are shown in Figs. $6 \mathrm{a}$ and $6 \mathrm{~b}$ for $g=9.8 \mathrm{~m} / \mathrm{s}^{2}$ and $p=5 \mathrm{~N} / \mathrm{m}$ with the presence of the micropolar thermoelasticity at $t=0.5 \mathrm{~s}$. These figures depict the dependence of these quantities on the distances $x$ and $y$ while they are moving during wave propagation.

(a)

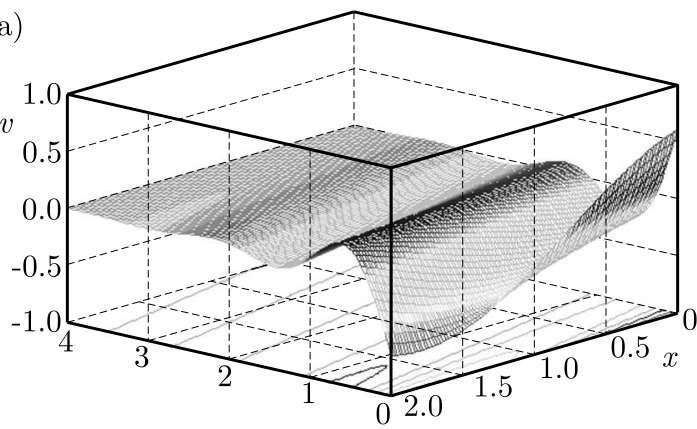

(b)

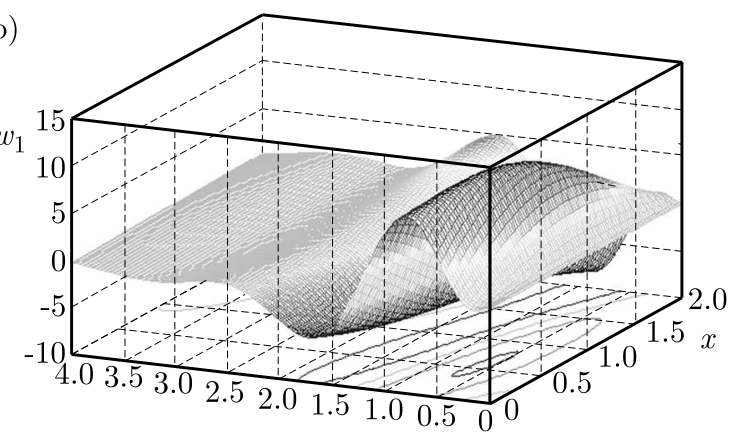

Fig. 6. Variation of displacement $v$ (a) and of microtemperature vector $w_{1}$ (b) versus distances $x$ and $y$

\section{Conclusion}

From the above analytical solutions, we conclude that:

1. Gravity and initial stress are effective physical factors having an important role in the variation of the physical quantities.

2. The micropolar thermoelasticity is an important property. The presence or the absence of this property is an observable effect in the variation of the considered physical quantities, for example in the variation of temperature.

3. The microtemperature is a very useful theory in the field of geophysics and earthquake engineering and for seismologists working in the field of mining tremors and drilling into the earth's crust. 
4. Values of all physical quantities converge to zero with an increase in the distance $y$, and all functions are continuous.

\section{Appendix A}

$$
\begin{array}{ll}
\psi_{1}(x, y, t)=\sum_{n=1}^{6} R_{n} \mathrm{e}^{-S_{n} y+\mathrm{i}(a x-\xi t)} & \psi_{2}(x, y, t)=\sum_{n=1}^{6} A_{1 n} R_{n} \mathrm{e}^{-S_{n} y+\mathrm{i}(a x-\xi t)} \\
q_{1}(x, y, t)=\sum_{n=1}^{6} A_{3 n} R_{n} \mathrm{e}^{-S_{n} y+\mathrm{i}(a x-\xi t)} & q_{2}(x, y, t)=\sum_{n=1}^{6} A_{4 n} R_{n} \mathrm{e}^{-S_{n} y+\mathrm{i}(a x-\xi t)} \\
w_{1}(x, y, t)=\sum_{n=1}^{6} G_{3 n} R_{n} \mathrm{e}^{-S_{n} y+\mathrm{i}(a x-\xi t)} & \sigma_{x x}(x, y, t)=\sum_{n=1}^{6} G_{5} n R_{n} \mathrm{e}^{-S_{n} y+\mathrm{i}(a x-\xi t)} \\
\sigma_{y y}(x, y, t)=\sum_{n=1}^{6} G_{6 n} R_{n} \mathrm{e}^{-S_{n} y+\mathrm{i}(a x-\xi t)} & \sigma_{x z}(x, y, t)=\sigma_{y z}(x, y, t)=0 \\
m_{y z}(x, y, t)=\sum_{n=1}^{6} G_{8 n} R_{n} \mathrm{e}^{-S_{n} y+\mathrm{i}(a x-\xi t)} & m_{x z}(x, y, t)=\sum_{n=1}^{6} G_{9 n} R_{n} \mathrm{e}^{-S_{n} y+\mathrm{i}(a x-\xi t)} \\
q_{x z}(x, y, t)=0 & q_{x x}(x, y, t)=\sum_{n=1}^{6} G_{10 n} R_{n} \mathrm{e}^{-S_{n} y+\mathrm{i}(a x-\xi t)} \\
q_{y y}(x, y, t)=\sum_{n=1}^{6} G_{11 n} R_{n} \mathrm{e}^{-S_{n} y+\mathrm{i}(a x-\xi t)} & q_{y z}(x, y, t)=0
\end{array}
$$

\section{Appendix B}

$$
\begin{aligned}
& N_{1}=c_{1}+1 \quad N_{2}=c_{9}+1 \quad N_{3}=a^{2}-\frac{c_{3} \xi^{2}}{N_{1}} \quad N_{4}=\frac{\mathrm{i} a c_{4}}{N_{1}} \\
& N_{5}=\frac{c_{3}}{N_{1}} \quad N_{6}=\mathrm{i} a c_{4} \quad N_{7}=a^{2}-c_{3} \xi^{2} \quad N_{8}=a^{2}+2 c_{5}-c_{7} \xi^{2} \\
& N_{9}=a^{2}+\frac{c_{11}-\mathrm{i} \xi c_{12}}{N_{2}} \quad N_{10}=\frac{c_{13}}{N_{2}} \quad N_{11}=-\mathrm{i} \xi c_{10} \quad N_{12}=a^{2}+c_{11}-\mathrm{i} \xi c_{12} \\
& N_{13}=a^{2}-\mathrm{i} \xi c_{14} \quad N_{14}=\mathrm{i} \xi c_{15} \quad A_{1 n}=\frac{S_{n}^{6}-l_{1} S_{n}^{4}+l_{2} S_{n}^{2}-l_{3}}{N_{4} S_{n}^{4}-l_{4} S_{n}^{2}+l_{5}} \\
& A_{2 n}=\frac{-N_{6}-A_{1 n}\left(S_{n}^{2}-N_{7}\right)}{c_{2}} \quad A_{3 n}=\frac{N_{10} A_{5 n}}{S_{n}^{2}-N_{9}} \quad A_{4 n}=\frac{-N_{11} A_{2 n}}{S_{n}^{2}-N_{12}} \\
& A_{5 n}=\frac{-N_{14} S_{n}^{4}+\left(N_{9} N_{14}+N_{14} a^{2}\right) S_{n}^{2}+N_{9} N_{14} a^{2}}{S_{n}^{4}-\left(N_{9}+N_{13}+c_{16} N_{10}\right) S_{n}^{2}+N_{9} N_{13}-c_{16} N_{10} a^{2}} \\
& A_{6 n}=c_{14}\left(\mathrm{i} a H_{1 n}-S_{n} H_{2 n}\right)+\mathrm{i} a c_{15} H_{1 n}-A_{5 n} \quad A_{9 n}=-c_{18} S_{n} A_{2 n} \\
& A_{7 n}=c_{14}\left(\mathrm{i} a H_{1 n}-S_{n} H_{2 n}\right)-S_{n} c_{15} H_{2 n}-A_{5 n} \quad A_{10 n}=\mathrm{i} a c_{18} A_{2 n} \\
& A_{8 n}=c_{16}\left(-S_{n} H_{1 n}+\mathrm{i} a H_{2 n}\right)+c_{17}\left(\mathrm{i} a H_{2 n}-A_{2 n}\right) \quad c_{1}=\frac{2(\lambda+\mu)+p}{2\left(\mu+k^{*}\right)-p} \\
& c_{2}=\frac{2 k^{*}}{2\left(\mu+k^{*}\right)-p} \quad c_{3}=\frac{2 \rho c_{0}^{2}}{2\left(\mu+k^{*}\right)-p} \quad c_{4}=\frac{2 \rho g c_{0}^{2}}{2\left(\mu+k^{*}\right)-p} \\
& c_{5}=\frac{2 k^{*} c_{0}^{2}}{\gamma \omega_{1}^{* 2}} \quad c_{6}=\frac{c_{0}^{2}\left(k^{*}-p\right)}{\gamma \omega_{1}^{* 2}} \quad c_{7}=\frac{\rho \mu_{1} c_{0}^{4}}{\gamma \gamma_{1} T_{0} \omega_{1}^{* 2}} \quad c_{8}=\frac{j \rho c_{0}^{2}}{\gamma}
\end{aligned}
$$




$$
\begin{aligned}
& c_{9}=\frac{k_{4}+k_{5}}{k_{6}} \quad c_{10}=\frac{\mu_{1} \gamma_{1} T_{0}}{\rho \omega_{1}^{*} k_{6}} \quad c_{11}=\frac{k_{2} c_{0}^{2}}{k_{6} \omega_{1}^{* 2}} \quad c_{12}=\frac{b c_{0}}{k_{6} \omega_{1}^{*}} \\
& c_{13}=\frac{k_{3} T_{0} c_{0}^{2}}{k_{6} \omega_{1}^{* 2}} \quad c_{14}=\frac{\rho C_{e} c_{0}^{2}}{k \omega_{1}^{*}} \quad c_{15}=\frac{\gamma_{1}^{2} T_{0}}{\rho k \omega_{1}^{*}} \quad c_{16}=\frac{k_{1}}{k T_{0}} \quad c_{17}=\frac{\lambda}{\rho c_{0}^{2}} \\
& c_{18}=\frac{2 \mu+k^{*}}{\rho c_{0}^{2}} \quad c_{19}=\frac{\mu+p}{2 \rho c_{0}^{2}} \quad c_{20}=\frac{2\left(k^{*}+\mu\right)-p}{2 \rho c_{0}^{2}} \quad c_{21}=\frac{k^{*}}{\rho c_{0}^{2}} \\
& c_{22}=\frac{\gamma \omega_{1}^{* 2}}{\rho c_{0}^{4}} \quad c_{23}=-k_{4} \mu \omega_{1}^{*} \quad c_{24}=-\left(k_{5}+k_{6}\right) \mu \omega_{1}^{*} \\
& c_{26}=-k_{6} \mu \omega_{1}^{*} \quad l_{1}=N_{3}+N_{9}+N_{13}-c_{16} N_{10}-N_{5} N_{14} \\
& l_{2}=N_{3}\left(N_{9}+N_{13}-c_{16} N_{10}\right)+N_{9} N_{13}-c_{16} N_{10} a^{2}-N_{5} N_{14}\left(N_{9}+a^{2}\right) \\
& l_{3}=N_{3}\left(N_{9} N_{13}-c_{16} N_{10} a^{2}\right)-N_{5} N_{9} N_{14} a^{2} \quad l_{4}=N_{4}\left(N_{9}+N_{13}-c_{16} N_{10}\right) \\
& l_{5}=N_{4}\left(N_{9} N_{13}-c_{16} N_{10} a^{2}\right) \quad G_{1 n}=\left(\mathrm{i} a-S_{n} A_{1 n}\right) \\
& G_{3 n}=\mathrm{i} a A_{3 n}-S_{n} A_{4 n} \quad G_{2 n}=-\left(S_{n}+\mathrm{i} a A_{1 n}\right) \\
& G_{4 n}=-\left(S_{n} A_{3 n}+\mathrm{i} a A_{4 n}\right) \quad c_{17}\left(\mathrm{i} a G_{1 n}-S_{n} G_{2 n}\right)+\mathrm{i} a c_{18} G_{1 n}-A_{5 n}-p \\
& G_{7 n}=-c_{19} S_{n} G_{1 n}+\mathrm{i} a c_{20} G_{2 n}-c_{21} A_{2 n}\left(\mathrm{i} a G_{1 n}-S_{n} G_{2 n}\right)-S_{n} c_{18} G_{2 n}-A_{5 n}-p \\
& G_{9 n}=\mathrm{i} a c_{22} A_{2 n} \quad G_{8 n}=-c_{22} S_{n} A_{2 n} \\
& G_{11 n}=c_{23}\left(\mathrm{i} a G_{3 n}-S_{n} G_{4 n}\right)-S_{n}\left(\mathrm{i} a G_{3 n}-S_{n} G_{4 n}\right)+\mathrm{i} a c_{24} G_{3 n} \\
& n=1,2, \ldots, 6 \quad G_{7 n}=-c_{15} S_{n} G_{3 n}+\mathrm{i} a c_{26} G_{4 n}
\end{aligned}
$$

\section{References}

1. Abbas I.A., Kumar R., 2013, Deformation due to thermal source in micropolar thermoelastic media with thermal and conductive temperatures, Journal of Computational and Theoretical Nanosciences, 10, 2241-2247

2. Abouelregal A.E., Zenkour A.M., 2015, Thermoelastic problem of an axially moving micro beam subjected to an external transverse excitation, Journal of Theoretical and Applied Mechanics, 53, 167-178

3. Ariman T., 1972, Wave propagation in a micropolar elastic half-space, Acta Mechanica, 13, 11-20

4. Ames K., Straughan B., 1999, Continuous dependence results for initially prestressed thermoelastic bodies, International Journal of Engineering Sciences, 30, 7-13

5. Bromwich T.J., 1898, On the influence of gravity on elastic waves and in particular on the vibrations of an elastic globe, Proceedings of the London Mathematical Society, 30, 98-120

6. Casas P.S., Quintanilla R., 2005, Exponential stability in thermoelasticity with microtemperatures, International Journal of Engineering Sciences, 43, 33-47

7. ERIngen A.C., 1966, Linear theory of micropolar elasticity, Journal of Mathematics and Mechanics, 15, 909-924

8. ERINGEn A.C., 1984, Plane wave in nonlocal micropolar elasticity, International Journal of Engineering Sciences, 22, 1113-1121

9. Eringen A.C., 1999, Microcontinuum Field Theory I: Foundations and Solids, Springer-Verlag, Berlin

10. Eringen A.C., Kafadar C.B., 1976, Polar field theories, [In:] Continuum Physics IV, Eringen A.C. (Edit.), Academic Press, New York

11. Grot R., 1969, Thermodynamics of continuum with microstructure, International Journal of Engineering Sciences, 7, 801-814 
12. IESAN D., 2001, On a theory of micromorphic elastic solids with microtemperatures, Journal of Thermal Stresses, 24, 737-752

13. IEsAn D., 2004, Thermoelastic Models of Continua, Springer Netherlands

14. IEsAn D., 2006, Thermoelasticity of bodies with microstructure and microtemperatures, International Journal of Solids and Structures, 43, 3414-3427

15. IESAN D., 2007, Thermoelasticity of bodies with microstructure and microtemperatures, International Journal of Solids and Structures, 44, 8648-8662

16. IEsan D., 2008, A theory of prestressed thermoelastic Cosserat continua, Journal of Applied Mathematics and Mechanics, 88, 306-319

17. IEsan D., Quintanilla R., 2000, On a theory of thermoelasticity with micro- temperatures, Journal of Thermal Stresses, 23, 199-215

18. Kumar R., Ailawalia P., 2005, Deformation in micropolar cubic crystal due to various sources, International Journal of Solids and Structures, 42, 5931-5944

19. Kumar R. Gupta R.R., 2010, Propagation of waves in transversely isotropic micropolar generalized thermoelastic half space, International Communications in Heat and Mass Transfer, 37, $1452-1458$

20. Love A.E.H., 1965, Some Problems of Geodynamics, Cambridge University Press, London

21. Montanaro A., 1999, On singular surfaces in isotropic linear thermoelasticity with initial stress, Journal of Acoustical Society of America, 106, 1586-1588

22. Othman M.I.A, Elmaklizi J.D., Saied S.M., 2013a, Generalized thermoelastic medium with temperature dependent properties for different theories under the effect of gravity field, International Journal of Thermophysics, 34, 521-537

23. Othman M.I.A., Hasona W.M., Abd-Elaziz E.M., 2014, Effect of rotation on micro-polar generalized thermoelasticity with two temperature using a dual-phase-lag model, Canadian Journal of Physics, 92, 148-159

24. Othman M.I.A, Zidan M.E.M., Hilal M.I.M., 2013b, The influence of gravitational field and rotation on thermoelastic solid with voids under Green-Naghdi theory, Journal of Physics, 2, 22-34

25. Othman M.I.A, Zidan M.E.M., Hilal M.I.M., 2015, The effect of initial stress on thermoelastic rotating medium with voids due to laser pulse heating with energy dissipation, Journal of Thermal Stresses, 38, 835-853

26. Parfitt V.R., Eringen A.C., 1971, Reflection of plane waves from a flat boundary of a micropolar elastic half-space, The Journal of Acoustical Society of America, 45, 1258-1272

27. Riha P., 1979, On the microcontinuum model of heat conduction in materials with inner structure, International Journal of Engineering Sciences, 14, 529-535

28. Scalia A., Svanadze M., 2006, On the representation of solutions of the theory of thermoelasticity with microtemperatures, Journal of Thermal Stresses, 29, 849-863

29. SEzAWA K., 1927, Dispersion of elastic waves propagated on the surface of stratified bodies and on curved surfaces, Bull Earthquake Research Institute Tokyo, 3, 1-18

30. Sмiтн A.C., 1967, Waves in micropolar elastic solids, International Journal of Engineering Sciences, 5, 741-746

31. Wang J., Slattery P., 2002, Thermoelasticity without energy dissipation for initially stressed bodies, International Journal of Mathematics and Mathematical Sciences, 31, 329-337 